\title{
Sollten Taucher Ohrentropfen mit Ciprofloxacin vorbeugend anwenden?
}

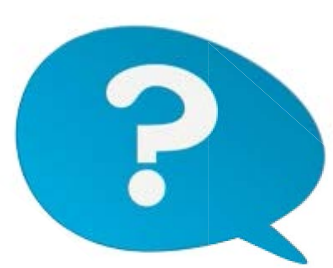

Frage: Wir untersuchen in unserer Praxis häufig Taucher und haben nun gehört, dass in den USA die prophylaktische Gabe von Ciprofloxacin-Ohrentropfen verbreitet ist. Ist eine derartige Prophylaxe sinnvoll? Gibt es hierzu Studien, die dieses Vorgehen unterstützen?

Antwort: Da die akute bakterielle Otitis externa in der überwiegenden Anzahl der Fälle durch Pseudomonas aeruginosa oder Staphylococcus aureus verursacht wird, ist die topische Anwendung von Ciprofloxacin-Ohrentropfen eine anerkannte Therapie bei einer korrekt diagnostizierten akuten Otitis externa. Generell muss bei der topischen Applikation von Antibiotika jedoch die potenzielle Gefahr einer allergenen Sensibilisierung in besonderer Weise berücksichtigt werden. Insofern erscheint diese Maßnahme nur bei einer klinisch gesicherten akuten Otitis externa gerechtfertigt. Der ungezielte Einsatz von CiprofloxacinOhrentropfen zur Prophylaxe bei Tauchern erscheint deshalb nicht empfehlenswert. Belastbare wissenschaftliche Studien zum Thema, die den prophylaktischen Einsatz von CiprofloxacinOhrentropfen bei Tauchern unterstützen, existieren unseres Wissens nach bislang nicht.

Statt der Anwendung antibiotischer Ohrentropfen sollten zur Prophylaxe

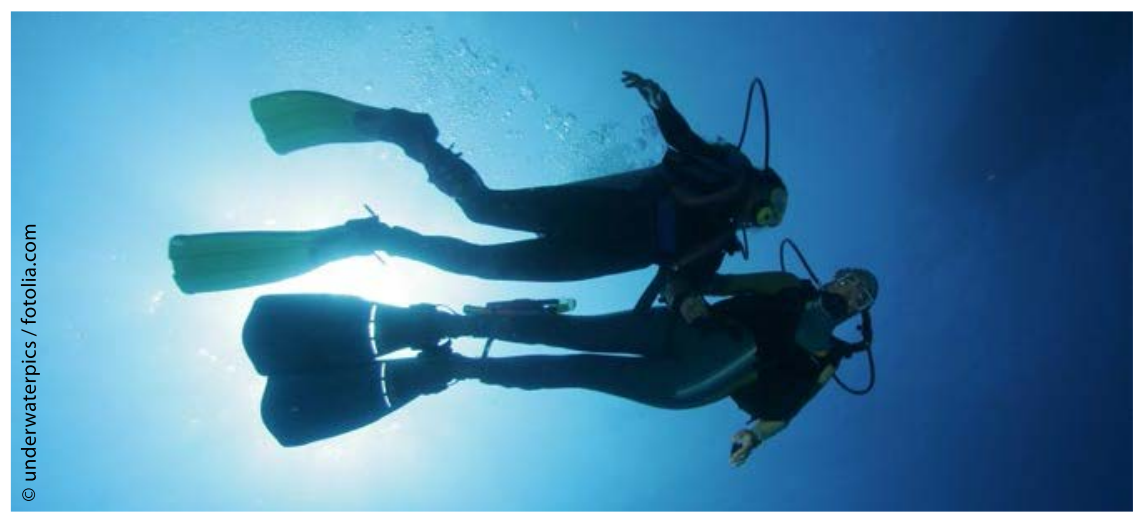

\section{Haben Sie auch eine fachliche Frage?}

Das "Consilium HNO" ist ein Service des Unternehmens Infectopharm. Als HNO-Arzt haben Sie die Möglichkeit, selbst Fragen zum gesamten Fachgebiet zu stellen. Schreiben Sie dazu an Dr. Kristin Brendel-Walter, Von-Humboldt-Straße 1, 64646 Heppenheim, oder schicken Sie eine E-Mail an consilium@infectopharm.com. Ihre Frage wird anonym an ein Mitglied des Expertenpools weitergeleitet, Sie erhalten eine schriftliche Antwort.

Wenn Sie sehen wollen, was die Kolleginnen und Kollegen so fragen, können Sie im Archiv des "Consilium HNO" unter www.infectopharm.com/consilium stöbern. Außerdem werden redaktionell ausgewählte, allgemein interessante Anfragen zusätzlich in der Zeitschrift „HNO-Nachrichten“ publiziert.

einer akuten Otitis externa bei Tauchern Maßnahmen Anwendung finden, die den bei dieser Gruppe bestehenden Prädispositionsfaktoren gezielt entgegenwirken.

Eine längerfristige beziehungsweise rezidivierende Exposition gegenüber Salzwasser kann zu einer Reduktion der schützenden Lipide des Cerumens im äußeren Gehörgang und zu einer Auflockerung des Epithels des äußeren Gehörgangs führen. Zusätzlich kommt es zu einer Erhöhung des protektiven sauren $\mathrm{pH}$-Wertes dieser Region.

Neben dem Ausspülen der Gehörgänge mit sauberem Süßwasser nach den Tauchgängen sollten deshalb pflegende, desinfizierende und den $\mathrm{pH}$ Wert wieder senkende Maßnahmen der Anwendung antibiotischer Ohrentropfen vorgezogen werden.

Als geeignete Prophylaxe bieten sich insbesondere Ohrentropfen mit den Bestandteilen Glycerol, Alkohol und Eisessig in unterschiedlichen Konzentrationen an (z. B. "Ehm'sche Lösung“" bestehend aus Eisessig 5,0 g, gereinigtem Wasser 10,0 g, 2-Propranolol [95 \%] 85,0 g oder „Heidelberger Ohrentropfen nach Klingmann" bestehend aus Glycerol 10,0 g, Ethanol [90 \%] ad $30,0 \mathrm{~g})$.

Lediglich für den Fall einer wirklich eingetretenen akuten Otitis externa sollte als Stand-by-Medikation über die Verordnung von CiprofloxacinTropfen nachgedacht werden.

\section{Dr. Jan Peter Thomas}

Universitätsklinikum Bochum

Klinik für Hals-Nasen- und Ohrenheilkunde, Kopf- und Hals-Chirurgie Bleichstraße 15 44787 Bochum 